\title{
Las competencias profesionales en Educación Física The professional skills in Physical Education
}

\author{
Clara Isabel Pazo Haro y Jesús Tejada Mora \\ Universitat de Huelva
}

\begin{abstract}
Resumen: El planteamiento inicial desde la aparición de los nuevos grados en la comunidad universitaria, tiene en común exigir a los estudiantes ir adquiriendo a lo largo de su formación, una serie de competencias que permitan alcanzar aquellos conocimientos, actitudes y destrezas: personales, profesionales y metodológicas, que se requieren para el desempeño de cada una de las profesiones. Aunque las competencias no son de obligado cumplimiento dentro de la antigua titulación de Magisterio de Educación Física en la Universidad de Huelva, nace la curiosidad por saber qué competencias y en qué grado se están desarrollando actualmente. De esta manera, los docentes universitarios tendrán una referencia más objetiva partiendo desde la realidad del alumnado, incidiendo en aquellas competencias que menos se desarrollan y reforzando las que más se favorecen en la actualidad. La metodología utilizada fue la cuantitativa, utilizando el cuestionario como instrumento de recogida de los datos y su posterior análisis a través del programa informático SPSS versión 18. La muestra estuvo formada por alumnos/as del último curso de Magisterio de Educación Física de la Universidad de Huelva, docentes del Área de Educación Física de dicha Universidad y maestros en activo de la especialidad señalada de la provincia de Huelva.
\end{abstract}

Palabra clave: formación, competencias, alumnado, Educación Física.

Abstract: The aim of the new degrees in the university community has in common to require students begin to acquire over their training, a range of skills to achieve the knowledge, attitudes and skills: personal, professional and methodological required for the performance of each of the professions. Although the skills are not mandatory in the old degrade of Teacher of Physical Education at the University of Huelva, one must know what skills and to what degree are currently being developed. Thus, university lecturers have a more objective reference starting from the reality of students, focusing on those skills that are less developed and strengthening the most favored ones today. The methodology used was quantitative, using the questionnaire as a measurement instrument and subsequent analysis through SPSS version 18 program. The sample was composed of pupils of the last year of teaching Physical Education at the University of Huelva, the lectures of Physical Education Area of the University and teachers in active of the province of Huelva

Key words: training, skills, students, Physical Education.

\section{Introducción}

Todos los seres humanos pueden mejorar su formación en cualquier ámbito. Para Gadner (2001) todo sujeto posee un total de ocho inteligencias que con esfuerzo pueden perfeccionarse. Estas inteligencias son: inteligencia lingüística, inteligencia lógica-matemática, inteligencia espacial, inteligencia musical, inteligencia corporal-cinestésica, inteligencia intrapersonal, inteligencia interpersonal e inteligencia naturalista. Teniendo como referencia estas inteligencias y con la intención de lograr en las personas su íntegro desarrollo, aparece en el ámbito educativo el término de competencias. En los estadíos de las enseñanzas superiores y dentro del proyecto Tuning se considera que las competencias son una buena referencia para poder unificar y comparar capacidades, que deberían poseer las personas que optan a un título académico, independientemente del lugar donde lo curse (Alonso, Fernández \& Nyssen, 2009). Por esta necesidad de equiparar los títulos dentro de los países miembros de la Comunidad Económica Europea nace la preocupación por conocer los requerimientos que deben tener los futuros profesionales para dominar y aplicar las destrezas, habilidades y conocimientos en un contexto en concreto.

El concepto de competencia tiene una amplia historia y no es por ello sorprendente que ser profesionalmente competente, ser suficientemente capaz y poder desempeñar ciertas tareas, haya sido una aspiración a lo largo de los tiempos. La competencia apareció en latín en la forma de competens que era concebido como el ser capaz y fue permitido por la ley/regulación, y en la forma de competentia, entendido como la capacidad y la permisión (Mulder, Wesselink \& Bruijstens, 2005). Alonso et al. (2009:23) definen el término competencia teniendo en cuenta diferentes organismos: La literatura contempla otras acepciones del término competencia (Perrenoud, 2004; Le Boterf, 1998; Aubert \& Gilbert, 2003; González \& Wagenaar, 2003; Cano, 2007), pero en

Fecha recepción: 12-11-11 - Fecha envío revisores: 12-11-11 - Fecha de aceptación: 07-12-11 Correspondencia: Clara Isabel Pazo Haro

Avda. Tres de Marzo s/n

21071 Huelva. España

E-mail: clara.pazo@uhu.es síntesis, se pueden extraer algunos elementos de interés. En el concepto se integra el saber, el saber hacer y el saber ser; tiene relación con la acción, se desarrolla y se actualiza en la acción; está vinculada a un contexto, a una situación dada y facilita la resolución eficaz de situaciones laborales conocidas o inéditas y es educable (Imbernón, Silva \& Guzmán, 2011). De estas definiciones se deduce que el término competencias engloba el desarrollo integral de las personas, no sólo se basa en aprendizajes teóricos. Hay que entender que el alumno es un ser humano que siente y que padece por lo que se debe establecer vínculos afectivos en donde a los alumnos se les acojan, se les comprendan y se confíen en sus posibilidades (Martín \& Puig, 2007).

El currículo de los grados trata de recoger todo esto, que en opinión de Zabalza (2003, p.62), no son más que una realidad viva porque se trata de algo que nace, que crece y se consolida, madura y se deteriora $y$ finalmente, muere y es sustituido por otro. Aunque esto no quita para reconocer que el Plan de Estudios es un punto clave de calidad pero no el único, porque debe ir acompañado de mejoras en las infraestructuras y organización institucional, la formación del profesorado, mejora de su estatus profesional y de apertura al exterior de la Universidad, etc.

Además el docente debe mejorar su formación en las competencias utilizando una metodología adecuada, creando situaciones que son complejas desde el principio. De ahí el cambio en la creación de saberes de la inteligencia humana, se trata por tanto, de crear problemas de complejidad creciente en la enseñanza superior (Perranaud, 2006). Para ello es importante que el profesor cambie su rol, teniendo en cuenta como dice Perranaud (2006, p.6) cuatro cambios: no considerar una relación pragmática con el saber como una relación menor, aceptar el desorden, lo incompleto, la aproximación de los saberes movilizados, como características inherentes a la lógica acción, despedirse del domino de la organización de los conocimientos en la inteligencia del alumno y tener una práctica personal de la utilización de los saberes en la acción.

Por ello, la implantación efectiva del Espacio Europeo de Educación Superior (EEES) ha supuesto la transformación notable de los estudios en Grados. Según expone el Documento Marco sobre la Integración del Sistema Universitario Español en el EEES, los objetivos formativos de las enseñanzas oficiales de nivel de grado tendrán, con carácter general, una orientación profesional, es decir, deberán propor- 
cionar una formación universitaria en la que se integren armónicamente las competencias genéricas básicas, las competencias transversales relacionadas con la formación integral de las personas y las competencias más específicas que posibiliten una orientación profesional que permitan a los titulados una integración en el mercado de trabajo (MECD, 2003).

En el EEES, una de las diversas clasificaciones de competencias existentes diferencia entre competencias genéricas y específicas. Las primeras son aquellas comunes y exigidas en todas las materias de la titulación, mientras que las específicas son las competencias que se pretenden fomentar y desarrollar en cada asignatura (Borrero \& Contreras, 2009). Según Corominas et al. (2006) las competencias específicas son saberes propios de una profesión específica (ej.: llevar a cabo una programación didáctica, gestionar una reserva a los clientes, calcular la resistencia de un forjado, etc. Las competencias genéricas son comunes a bastantes ámbitos profesionales (ej.: gestión de la información, adaptación a los cambios, disposición hacia la calidad, etc.). Las competencias específicas giran en torno al «saben» profesional, el «saber hacen» y el «saber guiar el hacer de otras personas»; y las competencias genéricas se ubican en el «saber estar» y el «saber ser».

Ante el inminente cambio de los planes de estudios dentro de la educación española superior y la nueva perspectiva que ello supone a través de las competencias profesionales, nace nuestra curiosidad por conocer las competencias profesionales más y menos desarrolladas durante la formación de los maestros de Educación Física de la Universidad de Huelva, según los estudiantes, profesores y egresados. Se analizará las diferencias de opinión sobre la adquisición de competencias actuales entre docentes, discentes y egresados.

\section{Metodología}

Esta investigación está dentro del paradigma positivista y es cuantitativa. La muestra estuvo formada por quince profesores del Área de Expresión Corporal de la Universidad de Huelva que imparten docencia en la carrera de Magisterio de Educación Física, setenta y cuatro alumnos de tercer curso de dicha diplomatura, y diecisiete egresados que han tenido su formación en dicha Universidad y han realizado la especialidad en Educación Física.

El instrumento de medida utilizado fue el cuestionario, que se dirigió a los profesores, alumnos y egresados de Huelva. Para la construcción del mismo se realizó un listado de categorías, agrupadas en dos dimensiones: competencias generales y competencias específicas:

Para la elaboración de los ítems de cada dimensión se utilizó una escala tipo Likert que consiste en un conjunto de ítems presentados en forma de afirmaciones o juicios ante los cuales se pide la reacción de los sujetos (Henández, Fernández \& Baptista, 2000, p.256). Se establecieron un total de 37 ítems, usando el nivel de medición ordinal, siendo 1 nada conforme, 2 Disconforme, 3 Más o menos conforme, 4 Conforme y 5 Totalmente conforme. El 0 es para no sabe no contesta.

El diseño provisional del cuestionario se estructuró en dos partes, la primera donde se solicita información general sobre la persona encuestada, y la segunda sobre las categorías a través de los ítems formulados.

El proceso de validación del cuestionario tuvo varias fases. En principio se presentó a un panel de expertos, constituidos por tres profesores de la Universidad de Huelva con alto conocimiento en metodología cuantitativa. A partir del consejo de los expertos se hizo una redacción provisional para la prueba piloto, incluyendo las modificaciones propuestas: las frases se pusieron en afirmativas y se introdujo el valor 0 para recoger el ítem «no sabe no contesta».

Se hizo una prueba piloto pasándoles el cuestionario a diez estudiantes de otro curso diferente al de la muestra, a diez profesores y a diez egresados de secundaria. Se calculó el índice de consistencia interna de los cuestionarios, a través del alfa de Cronbach obteniéndose un .944, siendo los valores entre 0 y 1 los adecuados para el nivel de relación de los diferentes ítems según Martín (2004).
Después se pasó a la elaboración definitiva del cuestionario y a su administración. El cuestionario se envió a través de correo electrónico a todos los profesores del Área de Educación Física de la Facultad de Ciencias de la Educación y después, se recogieron las contestaciones de todos los docentes excepto uno, a través de un casillero dispuesto para ello. A los estudiantes los datos se les tomaron en clase. Por último, la recogida de datos a los egresados de la provincia de Huelva, se hizo a través de un cuestionario electrónico y para los que trabajan en Huelva capital, se les entregó y recogió en su centro educativo. En total fueron diecisiete los egresados seleccionados, de un total de 50 que lo contestaron, tomando como muestra únicamente los que habían estudiado en la Universidad de Huelva y Magisterio por la especialidad de Educación Física.

\section{Resultados}

A continuación se muestran los datos obtenidos en el estudio, analizando por una parte la opinión de cada sector (alumnos, profesores y egresados) y, por otra, y las diferencias en cada ítem, entre cada uno de los grupos.

En la primera parte se toma como referencia el valor de la moda y después el de la media para saber qué competencias son las que más y menos están conformes en su desarrollo según cada sector. Y para la segunda parte, se comprueba las diferencias significativas intersectores, utilizando el anova factorial.

\subsection{Alumnos}

Para los alumnos las competencias generales que han adquirido con más afianzamiento durante su proceso de formación son las C6 (trabajar en equipo de forma cooperativa, para organizar y planificar el trabajo, tomando decisiones y resolviendo problemas, tanto de forma conjunta como individual), C9 (trabajar de forma colaborativa) y C15 (comportarse con ética y responsabilidad social como ciudadano y como profesional), teniendo una moda de 5 (totalmente de acuerdo). Dentro de las competencias específicas existen tres competencias en la que los alumnos están conforme de su adquisición a los largo de la carrera teniendo una moda de 4 y mayor media que las demás. Estas competencias son la $\mathrm{C} 17$ (comprender los principios que contribuyen a la formación cultural, personal y social desde la Educación Física), C23 (adquirir recursos para fomentar la participación a lo largo de la vida en actividades deportivas dentro y fuera de la escuela) y C37 (regular los procesos de interacción y comunicación en grupos de estudiantes 6 - 12 años).

Y la competencia general con la que más disconforme se encuentran los alumnos en su desarrollo es la C2 (expresarse de manera oral y escrita en varias lenguas (al menos en una lengua extranjera). Dentro de las competencias específicas con una moda de 3 , que expresa más o menos de acuerdo y una media menor que las demás, se encuentra la competencia C29 (conocer la evolución histórica del sistema educativo en nuestro país y los condicionantes políticos y legislativos de la actividad educativa).

\subsection{Profesores}

Dentro de las competencias generales los profesores están conformes con el desarrollo de la competencia C15 (comportarse con ética y responsabilidad social como ciudadano y como profesional), siendo su moda de 4 y media 3.57, la mayor de todas las categorías. Después con una moda igual, aunque con menor media se encuentran las competencias C9 (trabajar de forma colaborativa), C11 (leer e interpretar imágenes), y C6 (trabajar en equipo de forma cooperativa, para organizar y planificar el trabajo, tomando decisiones y resolviendo problemas, tanto de forma conjunta como individual). Con respecto a las competencias específicas se afirma que los docentes están conformes con el desarrollo durante la carrera de la competencia $\mathrm{C} 18$ (conocer el currículo escolar de la educación física), siendo su moda de 4 y su media de 3.79 . Otras competencias con la misma moda, pero con una media menor de 
3.64 son: la $\mathrm{C} 17$ (comprender los principios que contribuyen a la formación cultural, personal y social desde la Educación Física), C19 (adquirir un conocimiento práctico del aula y de la gestión de la misma), C25 (diseñar, planificar y evaluar la actividad docente y el aprendizaje en el aula) y C34 (relacionar teoría y práctica con la realidad del aula y del centro).

Y la competencia general C2 (expresarse de manera oral y escrita en varias lenguas (al menos en una lengua extranjera)) es la que en opinión de los profesores no han desarrollado en sus alumnos durante su formación. Y dentro de las competencias específicas los profesores están disconformes con el desarrollo de la competencia C29 (conocer la evolución histórica del sistema educativo en nuestro país y los condicionantes políticos y legislativos de la actividad educativa).

\subsection{Egresados}

Para los egresados las competencias generales que más destacan como adquiridas de manera más sólida durante sus estudios, con una moda de 5 y una media superior a 4, son la C9 (trabajar de forma colaborativa) y la C15 (comportarse con ética y responsabilidad social como ciudadano y como profesional). Dentro de las competencias específicas los egresados creen que las más desarrolladas fueron la $\mathrm{C} 17$ (comprender los principios que contribuyen a la formación cultural, personal y social desde la Educación Física) y C36 (participar en la actividad docente y aprender a saber hacer, actuando y reflexionando desde la práctica).

Sin embargo las competencias que menos han sido potenciadas en opinión de los egresados son: de manera general la C2 (expresarse de manera oral y escrita en varias lenguas (al menos en una lengua extranjera) con una moda de 1. Y específicamente la C29 (conocer la evolución histórica del sistema educativo en nuestro país y los condicionantes políticos y legislativos de la actividad edücativa) con una moda de $3, y$ la media más baja de 3.06 .

\subsection{Cruce de las opiniones de los sectores entrevistados}

En este apartado se realiza el cruce de datos entre las variables de las opiniones de los profesores, alumnos y egresados y el resto de ítems que conforma las distintas competencias. Se analiza las diferencias significativas existentes entre los sujetos del estudio, tomando como referencia los valores de anova factorial: si el índice es menor o igual de .05 se considera que existe una diferencia significativa entre las medias de los tres grupos. En la tabla se observa que no hay consenso al comparar la opiniones de los tres sectores, porque en veintidós competencias existen diferencias significativas entre ellos.

\begin{tabular}{|c|c|c|}
\hline \multicolumn{3}{|c|}{ Tabla 1. Diferencias signific ativas entre la variable competencia gen eral y cada sector } \\
\hline $\begin{array}{l}\text { COMPET ENCIAS } \\
\text { GENERALES }\end{array}$ & NOMBRE DE LA COMPETENCIA & $\begin{array}{c}\text { ANOVA } \\
\text { FACTORIAL }\end{array}$ \\
\hline $\mathrm{C} 7$ & $\begin{array}{l}\text { Criticar y ser creativo en el análisis, planificación y real ización de } \\
\text { tareas, como fru to de un pensamiento flexible y divergen te }\end{array}$ & .000 \\
\hline $\mathrm{C} 8$ & $\begin{array}{l}\text { Utilizar diversas fuentes de información, seleccionar, analizar, } \\
\text { sintetizar y extraer ideas importantes y gestionar la in formación }\end{array}$ & .000 \\
\hline C6 & $\begin{array}{l}\text { Trabajar en equipo de forma cooper ativa, para organizar y } \\
\text { planificar el trabajo, to mando decisiones y resolviendo problemas, } \\
\text { tanto de forma conjunta como individu a }\end{array}$ & .000 \\
\hline C9 & Trabajar de forma colaborativa & .000 \\
\hline $\mathrm{C} 4$ & Trabajar de forma autónoma con iniciativa & .002 \\
\hline $\mathrm{Cl}$ & Exponer las ideas elabo radas, de forma oral y en la escrita & .002 \\
\hline $\mathrm{C} 2$ & $\begin{array}{l}\text { Expresarte de manera oral y escrita en varias lenguas (al menos en } \\
\text { una lengua extranjera) }\end{array}$ & .011 \\
\hline $\mathrm{C} 3$ & Aprender a aprender & .011 \\
\hline $\mathrm{C} 15$ & $\begin{array}{l}\text { Comportarse con ética y responsabilidad social como ciudadano y } \\
\text { como profesional }\end{array}$ & 017 \\
\hline C5 & $\begin{array}{l}\text { Comprender los distintos códigos audiovisuales y multimedia y } \\
\text { manejo de las herramientas informáticas }\end{array}$ & . 028 . \\
\hline $\mathrm{C} 14$ & Aplicar un pensami ento crítico, lógico y creati vo & .035 \\
\hline $\mathrm{C} 10$ & Comunicarte de manera efectiva en un contorno de trabajo & .043 \\
\hline
\end{tabular}

Se comprueba que existen diferencias muy significativas en quince ítems, en las competencias generales, son nueve las competencias con este grado de diferencia $(\mathrm{C} 1, \mathrm{C} 2, \mathrm{C} 3, \mathrm{C} 4, \mathrm{C} 5, \mathrm{C} 6, \mathrm{C} 7, \mathrm{C} 8, \mathrm{C} 9, \mathrm{C} 10, \mathrm{C} 14$

\begin{tabular}{|c|l|c|}
\hline \multicolumn{3}{|c|}{ Tabla 2. Diferencias significativas entre la competen cia es pecífica y cada sector } \\
\hline $\begin{array}{c}\text { COMPETENCIAS } \\
\text { ESPECÍFICAS }\end{array}$ & \multicolumn{1}{|c|}{ NOMBRE DELA COMPETENCIA } & $\begin{array}{c}\text { ANOVA } \\
\text { FACTORIAL }\end{array}$ \\
\hline C31 & Abordar y resolver problemas de disciplina & .000 \\
\hline C30 & Conocer los procesos de interacción y comunicación en el aula & .000 \\
\hline C16 & $\begin{array}{l}\text { Desarrollar y evaluar contenidos del currículo mediante recursos } \\
\text { didátcicos apropiados y promover la adquisición de competencias } \\
\text { básicas en los estudiantes }\end{array}$ & .005 \\
\hline C29 & $\begin{array}{l}\text { Conocer la evol ución histórica del si sema educativo en nuestro país } \\
\text { ylos condicionantes políticos y legi slativos de la actividad educativa }\end{array}$ & .005 \\
\hline C32 & Conocer yapli car experiencias innovadoras en educación pri maria & .008 \\
\hline C26 & $\begin{array}{l}\text { Relacionar la edu cación con el medio, y cooperar con las familias y } \\
\text { lacomunidad }\end{array}$ & .012 \\
\hline C28 & $\begin{array}{l}\text { Analizar la práctica docente y las condiciones institucionales que la } \\
\text { enmarcan }\end{array}$ & .021 \\
\hline C27 & $\begin{array}{l}\text { Regular los procesos de interacción y comunicación en grupos de } \\
\text { estudiantes 6 -12 años }\end{array}$ & .021 \\
\hline C27 & $\begin{array}{l}\text { Conocer y aplicar los procesos de interacción y comunicación en el } \\
\text { aula y dominar las destrezas y habilidades sociales necesarias para } \\
\text { fomentar un clima de aula que facilite el aprendizaje y la } \\
\text { convivencia }\end{array}$ & .021 \\
\hline & $\begin{array}{l}\text { Identificar y planificar la resolución de situaciones educativas que } \\
\text { afectana estudiantes con diferentes capacidades y distintos ritmos de } \\
\text { aprendizaje }\end{array}$ & .035 \\
\hline
\end{tabular}

y C15). Y dentro de las competencias específicas destacaría por su gran diversidad seis de ellas (C16, C20, C26, C27, C28, C29, C30, C31, C32 y C37).

\section{Discusión}

En general, Perrenaud (2006) aboga por la introducción de estas competencias dentro de las diferentes materias, ya que opina que se debe tender a una menor separación entre las disciplinas de manera que se acepten que hay una parte de sus funciones menos centradas en la materia y más en los alumnos. Para ello, es esencial el conocimiento del currículo para saber responder a las circunstancias, condiciones e intereses concretos de las personas, aunque sean mudables con el tiempo (Zabalza, 2003).

En los resultados se muestran como las competencias de trabajo en grupo y de manera colaborativa (C6 y C9) son fundamentales para la formación de los maestros de Educación Física. En opinión de Martín y Puig (2007) tiene ventajas como el compartir responsabilidades, aumentar la reflexión y el análisis colectivo, intervenciones más globales, etc. En el estudio Corominas et al. (2006) existen un grupo de competencias consideradas por ellos como núcleo de interés formativo, las cuales son: las relaciones interpersonales, trabajo en equipo, toma de decisiones y solución de problemas, constituyen un núcleo de interés según la opinión del profesorado. Sin embargo según Mora (2003) las competencias menos adquiridas por los egresados durante sus carreras eran las que giraban en torno a la participación: capacidad de liderazgo, tomar decisiones, asumir responsabilidades y, en cambio en la universidad española: el trabajo en equipo coincide con nuestro trabajo en ser destacado, además de la confianza en sí mismo, la capacidad crítica y la rigurosidad en el trabajo

Según el estudio las competencias que menos han sido potenciadas son: de manera general la C2 (expresarse de manera oral y escrita en varias lenguas (al menos en una lengua extranjera), y específicamente la C29 (conocer la evolución histórica del sistema educativo en nuestro país y los condicionantes políticos y legislativos de la actividad educativa). Sin embargo, los datos de Alonso et al. (2009) discrepan con los del estudio porque para los egresados de su investigación, la carencia estaría más en la aplicación de los conocimientos de la carrera, no tanto en la falta de conceptos históricos como en este caso. Además las competencias deben tener una relación directa con la práctica deportiva, y el maestro debe posibilitar un buen clima de trabajo afianzando competencias referidas a actitudes, tan importante en las características de un buen profesor como afirma Zabalza (2003).

\section{Conclusiones}

Todos los sectores concluyen que las competencias generales C15, con respecto al tema social y ciudadano, C9 en relación al trabajo colaborativo y la específica C17 centrándose en aspectos de la Educa- 
ción Física, son las más desarrolladas durante la carrera universitaria. Sin embargo, la competencia $\mathrm{C} 2$ relacionada con el aprendizaje de idiomas ha tenido menos adquisición durante la formación de los discentes.

Por otro lado, se observa que hay un escaso acuerdo entre los distintos sectores. Los profesores están más disconformes con respecto al grado de consecución de las competencias en general, mientras que los alumnos tienden a estar más conformes con el afianzamiento de las mismas y la opinión de los egresados es más central.

\section{Referencias}

Alonso, L. E.; Fernández, C. J., \& Nyssen, J. M. (2009). El debate sobre las competencias. Una investigación cualitativa en torno a la educación superior y el mercado de trabajo en España. Madrid: ANECA.

Aubert, J., \& Gilbert, P.(2003).L'évaluation des compétences. Sprimont, Bélgica: Mardaga.

Borrerro, C., \& Contreras, L. C. (2009). Evaluación de competencias en entornos virtuales. En Aguaded, J. I., y Fonseca M. C. (Ed.) Huellas de innovación docente en las aulas universitarias, pp.77-86. La Coruña: Netbiblo.

Cano, E. (2007). Cómo mejorar las competencias de los docentes. Guía para la autoevaluación y el desarrollo de las competencias del profesorado. Barcelona: Graó.

Corominas, E., Tesouro, M., Capell, D., Teixidó, J., Pèlach, J., \& Cortada, R. (2006). Percepciones del profesorado ante la incorporación de las competencias genéricas en la formación universitaria. Revista de Educación, 341,301-336.

Fernández, C., \& Baptista, P. (2000). Metodología de la investigación. México: MCGraw-Hill Interamericana.
Gardner, H. (2001). Estructura de la mente e inteligencia múltiples. $6^{\mathrm{a}}$ edición. Colombia: Editorial Mac Graw Hill

Gardner, H. (2001). Estructura de la mente e inteligencia múltiples. $6^{\mathrm{a}}$ edición. Colombia: Editorial Mac Graw Hill.

González, J., \& Wagenaar, R. (Coord.) (2003). Tuning Educational Structures in Europe. Informe Final. Bilbao: Universidad de Deusto.

Hernández, R.; Fernández, C., \& Baptista, P. (2000). Metodología de la investigación. México: MCGraw-Hill Interamericana.

Imbernón, F., Silva P., \& Guzmán C. (2011). Teaching Skills in Virtual and Blended Learning. Environments, 36, 107-114.

Le Boterf, G (1998). La ingeniería de las competencias. D'organisation, 6;23-33.

Martín, M. C. (2004). Diseño y validación del cuestionarios. Matronas Profesión, 5, 17, 23-29.

Martín, X., \& Puig, J. M. (2007). Las siete competencias básicas para educar en valores. Barcelona: Grao.

MECD (2003). La integración del sistema universitario en el Espacio Europeo de Educación Superior. Documento-Marco. Madrid: Ministerio de Educación, Cultura y Deporte (Eds.).

Mora, J. G. (2003). Competencias y empleo de los jóvenes graduados universitarios. Revista de Educación, 330, 157-170.

Mulder, M., Wesselink, R., \& Bruijstens, H. Chr. J. (2005). «Job profile research for the purchasing profession». International Journal of Training and Development, 9(3), 185-204.

Perrenaud, P. (2006). Construir competencias desde la Escuela. Santiago: Ediciones Noreste.

Perrenoud, P. (2004). Diez nuevas competencias para enseñar. Barcelona: Graó.

Zabalza, M. A. (2003). Competencias docentes del profesorado universitario.Calidad y desarrollo profesional. Madrid: Nancea.

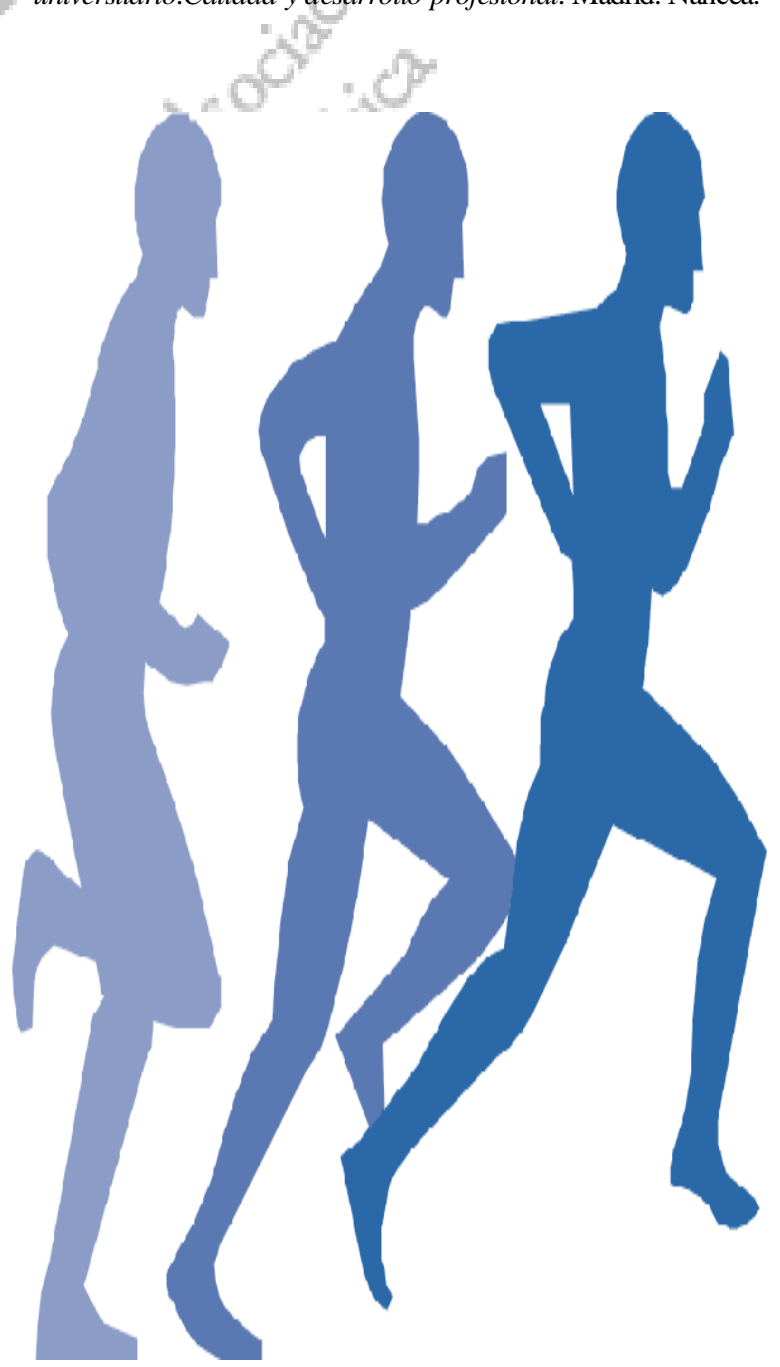

